\title{
Loss of AMP-Activated Protein Kinase Induces Mitochondrial Dysfunction and Proinflammatory Response in Unstimulated Abcd1-Knockout Mice Mixed Glial Cells
}

\author{
Jaspreet Singh, Hamid Suhail, and Shailendra Giri \\ Department of Neurology, Henry Ford Health System, Detroit, MI 48202, USA \\ Correspondence should be addressed to Jaspreet Singh; jsingh2@hfhs.org
}

Received 12 January 2015; Accepted 18 February 2015

Academic Editor: Luc Vallières

Copyright (C) 2015 Jaspreet Singh et al. This is an open access article distributed under the Creative Commons Attribution License, which permits unrestricted use, distribution, and reproduction in any medium, provided the original work is properly cited.

\begin{abstract}
$\mathrm{X}$-linked adrenoleukodystrophy (X-ALD) is caused by mutations and/or deletions in the ABCD1 gene. Similar mutations/deletions can give rise to variable phenotypes ranging from mild adrenomyeloneuropathy (AMN) to inflammatory fatal cerebral adrenoleukodystrophy (ALD) via unknown mechanisms. We recently reported the loss of the anti-inflammatory protein adenosine monophosphate activated protein kinase (AMPK $\alpha 1$ ) exclusively in ALD patient-derived cells. X-ALD mouse model (Abcd1knockout (KO) mice) mimics the human AMN phenotype and does not develop the cerebral inflammation characteristic of human ALD. In this study we document that AMPK $\alpha 1$ levels in vivo (in brain cortex and spinal cord) and in vitro in Abcd1-KO mixed glial cells are similar to that of wild type mice. Deletion of AMPK $\alpha 1$ in the mixed glial cells of Abcd1-KO mice induced spontaneous mitochondrial dysfunction (lower oxygen consumption rate and ATP levels). Mitochondrial dysfunction in ALD patient-derived cells and in AMPK $\alpha 1$-deleted Abcd1-KO mice mixed glial cells was accompanied by lower levels of mitochondrial complex (1-V) subunits. More importantly, AMPK $\alpha 1$ deletion induced proinflammatory inducible nitric oxide synthase levels in the unstimulated Abcd1-KO mice mixed glial cells. Taken together, this study provides novel direct evidence for a causal role for AMPK loss in the development of mitochondrial dysfunction and proinflammatory response in X-ALD.
\end{abstract}

\section{Introduction}

$\mathrm{X}$-linked adrenoleukodystrophy (X-ALD) is an inherited neuroinflammatory demyelinating peroxisomal disorder [1]. The underlying defect is a mutation/deletion in the ABCD1 gene that encodes the peroxisomal integral membrane transporter adrenoleukodystrophy protein (ALDP) [2]. ALDP is responsible for importing very long chain fatty acids (VLCFA; $\mathrm{C}>22: 0$ ) into the peroxisomes for degradation, a function exclusive to peroxisomes. As a result, VLCFA accumulate in the tissues and body fluids of X-ALD patients, a biochemical hallmark of the disease [3]. The disease has two major phenotypes: severe inflammatory and often fatal cerebral adrenoleukodystrophy (ALD) and mild relatively benign adrenomyeloneuropathy (AMN) $[1,3]$. ALD patients develop spontaneous neuroinflammatory responses and demyelination, which results in death within 2-5 years from the onset of symptoms [1]. AMN patients, on the other hand, live into adulthood with mild axonopathy [1]. However, about $30 \%$ of AMN patients progress spontaneously to fatal ALD phenotype in adulthood [1]. The mechanism(s) for differential phenotypes (AMN or ALD) or the progress of AMN to ALD phenotype remain unknown $[1,4]$. Intriguingly, the ABCD1 mutation and VLCFA levels are common among the two major phenotypes [4]. In fact, both the phenotypes are detectable within a family with similar ABCD1 mutations; thus, there is no phenotype-genotype correlation [1]. An animal model of X-ALD, a classical knockout of Abcdl (Abcd1-KO), accumulates VLCFA in tissues and body fluids but fails to develop the neuroinflammatory response [57]. In the late stage of life (>15 months), the mice develop axonopathy in the spinal cord and thus the mouse model at best resembles the human AMN phenotype [8].

We recently documented the first evidence of loss of a metabolic gene, AMP-activated protein kinase (AMPK), in ALD but not AMN patient-derived cells [9]. In vivo 
and in vitro studies have shown that AMPK signaling and proinflammatory responses are mutually coupled via negative feedback [10-12]. Activation of AMPK suppresses proinflammatory mediators $[13,14]$ while stimulation with inflammatory cytokines promotes dephosphorylation and hence inhibition of AMPK [14]. ALD patient-derived cells lacking AMPK demonstrated an increased proinflammatory gene expression [9]. Mitochondrial dysfunction (measured as oxygen consumption rate (OCR)) was also observed in ALD patient-derived cells [9]. This was not surprising considering that AMPK is the principal upstream regulator of mitochondrial function and loss of AMPK induces spontaneous mitochondrial dysfunction and proinflammatory response both in vivo and in vitro [12, 15]. A direct causal role for AMPK $\alpha 1$ in the X-ALD neuroinflammatory response, however, remained to be investigated. The status of AMPK $\alpha 1$ in Abcdl-KO mice central nervous systems is unknown. Since Abcd1-KO mice mimic the human AMN phenotype [5-7] and do not develop the cerebral inflammation characteristic of human ALD [5-8], in this study we investigated the status of AMPK $\alpha 1$ in the brains and spinal cords of Abcd1-KO mice. Furthermore, the expression and levels of AMPK $\alpha 1$, mitochondrial complex subunits, and mitochondrial OCR were compared between wild type (WT) and Abcd1-KO mice mixed glial cells. To investigate a causal role for AMPK $\alpha 1$ in the development of the neuroinflammatory response in $\mathrm{X}$-ALD, we used lentiviral vector carrying mouse AMPK $\alpha 1$ shRNA to delete AMPK $\alpha 1$ in Abcd1-KO mouse primary mixed glial cells. Mitochondrial function (OCR) and induction of proinflammatory response were compared between WT, Abcd1-KO, and AMPK $\alpha 1$-deleted Abcd1-KO mice mixed glial cells.

\section{Materials and Methods}

2.1. Reagents. Dulbecco's Modified Eagle's Medium (DMEM, $4.5 \mathrm{~g} / \mathrm{L}$ ) and fetal bovine serum were purchased from BioAbChem Inc. (Ladson, SC). Antibodies were purchased from Cell Signaling Technology Inc. (Boston, MA), unless otherwise mentioned. ECL and nitrocellulose membranes were purchased from BioRad (Hercules, CA). MitoProfile Total OXPHOS WB Antibody Cocktail was purchased from Abcam (Cambridge, MA). ATP determination kit was from Molecular Probes (Invitrogen, Grand Island, NY).

2.2. Abcd1-Knockout (Abcd1-KO) Mice. Abcd1-KO mice were obtained from Jackson Laboratories and housed in the pathogen-free animal housing facility of the Henry Ford Health System. Animal procedure (\#1271) was approved by the Henry Ford Health System Animal Review Committee, and all animals received humane care in compliance with the institution's experimental guidelines (Guide for the Care and Use of Laboratory Animals).

\subsection{Cell Culture}

2.3.1. Fibroblasts. Human skin fibroblasts derived from healthy control (CTL1, GM03348, CTL2, and GM03377),
ALD (ALD1, GM04934, ALD2, and GM04904), and AMN (AMN1, GM07531, AMN2, and GM17819) patients were obtained from the National Institute of General Medical Sciences Human Genetic Cell Repository (https://catalog.coriell .org/) and cultured as described previously [9].

2.3.2. Primary Mixed Glial Cells. Mouse primary mixed glial cells were prepared from 2-day-old WT and Abcd1-KO pups, as described previously [16].

2.4. Lentiviral Vector Mediated Knockdown of AMPKa1 in Abcd1-KO Mice Mixed Glial Cells. Transduction-ready mouse shRNA lentiviral particles $\left(10^{6} \mathrm{TU} / \mathrm{mL}\right)$ for AMPK $\alpha 1$ (consisting of a pool of 3-5 constructs and puromycin selection gene; sc-29674-V) and control shRNA lentiviral particles (Scr) $\left(10^{6} \mathrm{TU} / \mathrm{mL}, \mathrm{sc}-108080\right)$ were purchased from Santa Cruz Biotechnology (Dallas, TX).

Abcd1-KO mixed glial cells were cultured in DMEM with $10 \%$ fetal bovine serum, and viral particles (AMPK $\alpha 1$ and control) were added with a multiplicity of infection of 2.5 . Transduced cells were selected using puromycin $(3.0 \mu \mathrm{g} / \mathrm{mL})$. AMPK $\alpha 1$ silencing was observed by western blot and mRNA quantification.

2.5. Western Blot Analysis. Samples for western blot were prepared and ran as described previously [9]. The membranes were probed with AMPK $\alpha 1$, PGC- $1 \alpha$ (Santa Cruz Biotechnology, Dallas, TX), or MitoProfile Total OXPHOS Rodent WB Antibody Cocktail. The membranes were detected by autoradiography using ECL-plus.

2.6. RNA Extraction and cDNA Synthesis. Following total RNA extraction using TRIzol (Invitrogen), per the manufacturer's protocol, single-stranded cDNA was synthesized from $5 \mu \mathrm{g}$ of total RNA using iScript cDNA synthesis kit (BioRad, Hercules, CA).

2.7. Real-Time Polymerase Chain Reaction. Real-time polymerase chain reaction (PCR) was conducted using Bio-Rad's CFX96 Real-Time PCR Detection System. The primer sets for use were synthesized from Integrated DNA Technologies (Coralville, IA). IQ SYBR Green Supermix was purchased from Bio-Rad. The normalized expression of target gene with respect to L27 was computed for all samples.

2.8. Measurement of Mitochondrial Oxygen Consumption and Extracellular Flux. Oxygen consumption in intact adherent WT and Abcdl-KO mixed glial cells was measured using a Seahorse Bioscience $\mathrm{XF}^{\mathrm{e}} 96$ Extracellular Flux Analyzer (North Billerica, MA), as previously described [9]. Mixed glial cells were seeded to $1.5 \times 10^{4}$ cells/well in $\mathrm{XF}^{\mathrm{e}}$ 96-well cell culture microplate (Seahorse Bioscience) in $200 \mu \mathrm{L}$ of DMEM and cultured at $37^{\circ} \mathrm{C}$ in $5 \% \mathrm{CO}_{2}$ atmosphere. The growth medium was replaced with $175 \mu \mathrm{L}$ of bicarbonatefree DMEM, and the cells were incubated for 1 hour for degassing before starting the assay procedure. Basal 
and carbonyl cyanide p-trifluoromethoxyphenylhydrazone(FCCP-) linked OCR was measured as described by us previously [9].

2.9. Determination of ATP Levels. Primary Abcd1-KO mice mixed glial cells $\left(2 \times 10^{4}\right.$ cells/well $)$ were seeded in a 96well cell culture plate in complete medium and deleted for AMPK $\alpha 1$ as described above. Cells were lysed in $20 \mu \mathrm{L}$ lysis buffer and $10 \mu \mathrm{L}$ of lysate was used to measure ATP levels using an ATP determination kit (Molecular Probes, Invitrogen). $1 \mu \mathrm{L}$ of the cell lysate was used for normalization of protein levels.

2.10. Statistical Analysis. Using the Student Newman-Keuls test and analysis of variance, $P$ values were determined for the respective experiments from three identical experiments using GraphPad software (GraphPad Software Inc., San Diego, CA). The criterion for statistical significance was $P<$ 0.05 .

\section{Result and Discussion}

3.1. AMPK $\alpha 1$ Levels Are Similar between WT and Abcd1KO Mice Brains and Mixed Glial Cells. The mechanism(s) of induction of the neuroinflammatory response in X-ALD remains unknown $[1,4]$. More intriguing is the observation that the inflammatory response and demyelination have no genotype correlation $[1,4]$. Individuals with the same ABCD1 mutation (even between monozygotic twins) may develop strikingly opposite phenotypes of fatal neuroinflammatory ALD (and hence a shortened life span) or relatively benign AMN phenotype that exhibits only mild spinal cord pathology that is too late in adulthood $[1,3]$. We recently documented the first evidence of differential loss of a metabolic and anti-inflammatory protein, AMPK $\alpha 1$, in the patient-derived fibroblasts and lymphocytes of the severe (ALD) phenotype of X-ALD [9]. AMPK $\alpha 1$ levels between healthy control and AMN patient-derived cells were largely unchanged [9]. Our laboratory previously reported that loss of AMPK $\alpha 1$ is associated with more severe neuroinflammation and neurodegeneration in an animal model of multiple sclerosis [17]. Abcd1-KO mice fail to develop the neuroinflammation and demyelination characteristic of human ALD and only mimic the human mild AMN phenotype [5-8]. It was, therefore, of interest to investigate the status of AMPK $\alpha 1$ in Abcd1-KO mice brains and spinal cords. In line with our recent report in human AMN patient-derived cells (compared to healthy controls) [9], there was no significant difference in AMPK $\alpha 1$ protein and mRNA levels in the brain cortexes or spinal cord of age-matched (3-month-old) Abcd1KO mice compared with WT mice (Figure 1(a)).

Also, AMPK $\alpha 1$ levels in the primary mixed glial cells of Abcd1-KO mice were not decreased and were, in fact, higher than those in WT mice mixed glial cells (Figure 1(b)). In both cells and animal models with AMPK deletion, there is spontaneous mitochondrial dysfunction and proinflammatory response $[10,12,18,19]$. To investigate if AMPK $\alpha 1$ loss in Abcd1-KO mice mixed glial cells can induce mitochondrial dysfunction and inflammation reminiscent of the ALD phenotype, Abcd1-KO mixed glial cells were silenced for AMPK $\alpha 1$ using lentiviral-shRNA (Figure 1(b)). Following transduction and selection of transduced cells with puromycin, lentiviral-mediated silencing of AMPK $\alpha 1$ in Abcd1-KO mice mixed glial cells was highly successful (Figure 1(b)). Western blot analysis showed complete loss of AMPK $\alpha 1$ protein in Abcd1-KO mixed glial cells silenced for AMPK $\alpha 1$ (Figure 1(b)(i)). Real-time PCR with primers against mouse AMPK $\alpha 1$ showed significant $(P<0.001)$ downregulation of AMPK $\alpha 1$ expression (Figure 1(b)(ii)). Silencing with nontargeting scrambled sequence (Scr) had no effect on the levels of AMPK $\alpha 1$, thereby suggesting that lentiviral vector-mediated knockdown in Abcdl-KO mixed glial cells was specific for AMPK $\alpha 1$.

3.2. Mitochondrial Complex (I-V) Gene Expression and Levels Are Reduced in ALD Patient-Derived Cells and in Abcd1KO Mixed Glial Cells Deleted for AMPK $\alpha 1$. There is increasing evidence that mitochondrial dysfunction is important in the pathophysiology of X-ALD [20, 21]; however, the exact upstream mechanism(s) remain hypothetical at this point [22]. AMPK is the principal upstream regulator of mitochondrial biogenesis and function [15]. We documented the first report that $\mathrm{AMPK} \alpha 1$ levels and mitochondrial function (OCR), a measure of oxidative phosphorylation (OXPHOS), were decreased in ALD patient-derived cells [9]. The OXPHOS system is composed of five complexes, four of which, complexes I-IV, cooperate to generate a proton gradient across mitochondrial inner membrane. Complex V generates the universal energy ATP coupling with proton flow [23]. Quantitative PCR (Figure 2(a)) for individual subunits comprising complexes I-V and antibody cocktail against all five complexes showed (Figure 2(b)) that mitochondrial complex subunit expressions and levels were also significantly decreased in ALD patient-derived cells when compared to AMN patient-derived cells. Two subunits of complex I (NDUFS8 and NDUFB1) and 1 of complex II (SDHA) were significantly $(P<0.05)$ reduced in AMN fibroblasts when compared to control healthy patient-derived fibroblasts, while the rest of the subunit expressions were unchanged between AMN and healthy control patient-derived cells (Figure 2(a)).

Similar to human AMN cells (compared to healthy controls) (Figure 2(a)), mitochondrial complex subunit expressions and levels in Abcd1-KO mice mixed glial cells were comparable to control WT mice mixed glial cells (Figure 3(a)). This could be expected since AMPK $\alpha 1$ levels were unchanged between Abcd1-KO mice's central nervous systems and mixed glial cells (Figure 1(b)). Moreover, Abcd1-KO mice mimic human AMN phenotype [8]. This provided us with an opportunity to test if deletion of AMPK $\alpha 1$ in Abcd1-KO mixed glial cells could mimic changes in mitochondrial complex expressions similar to that found in ALD patientderived cells (Figure 2). Abcd1-KO mice mixed glial cells deleted for AMPK $\alpha 1$ indeed had significantly reduced expression (Figure 3(a)) and protein levels (Figure 3(b)) of mitochondrial complex subunits. Although their individual role 


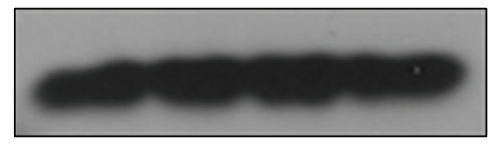
$\mathrm{AMPK} \alpha 1$

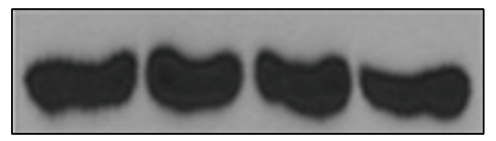

(i)

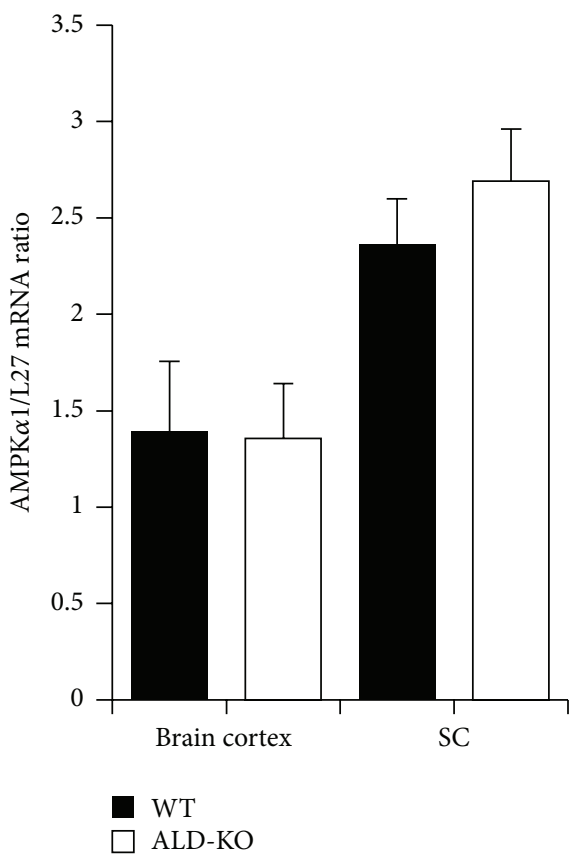

(ii)
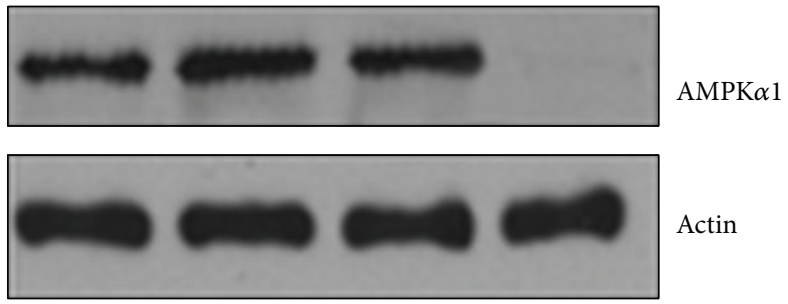

(i)

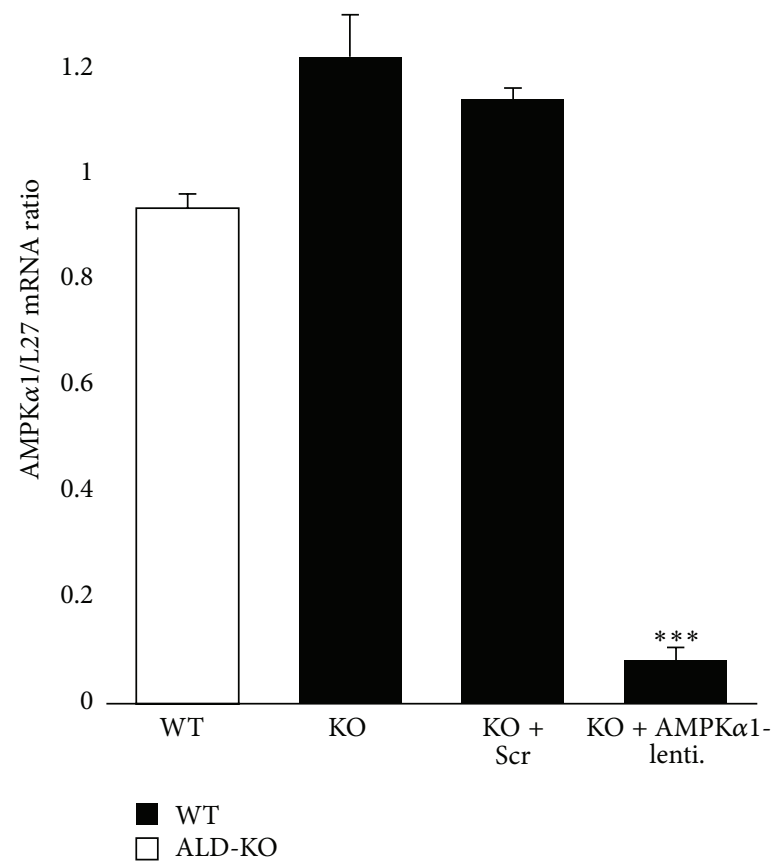

(ii)

(b)

FIGURE 1: Adenosine monophosphate activated protein kinase (AMPK $\alpha 1$ ) levels are similar in vivo in wild type (WT) and Abcd1-knockout (KO) central nervous systems and in vitro in mixed glial cells. (a) Age-matched (3-month-old) WT and Abcdl-KO mice were sacrificed and the brains and spinal cords harvested for AMPK $\alpha 1$ protein (i) and mRNA (ii) levels. (b) In primary mixed glial cells from WT and Abcd1KO mice, AMPK $\alpha 1$ protein (i) and mRNA (ii) levels were similar. (b) Abcdl-KO mixed glial cells were silenced for scrambled control (Scr) or AMPK $\alpha 1$ as described in Section 2. Lentiviral vector silencing with AMPK $\alpha 1$-shRNA significantly decreased the AMPK $\alpha 1$ protein (i) and mRNA (ii) levels in Abcd1-KO mice primary mixed glial cells. Results represent the mean \pm SD of triplicates from three different experiments. ${ }^{* * *} P<0.001$ AMPK $\alpha 1$ silenced compared with scrambled (Scr) silenced.

in X-ALD remains to be investigated, underexpression of multiple mitochondrial subunits has been associated with neurodegenerative conditions [24]. For instance, Leigh's syndrome is a severe neurodegenerative disease associated with mutations and underexpression of mitochondrial complexes [25-28]. Mutations and underexpression of multiple complex I subunits (including NDUFS1 and NDUFS8 observed here in ALD fibroblasts/AMPK $\alpha 1$-deleted Abcd1-KO cells) are associated with severe Leigh's syndrome [25-27]. Homozygous mutations in the complex II subunit SDHA gene are also associated with Leigh's syndrome [28]. Complex II is critical to mitochondrial function since it lies at the crosspoint of OXPHOS and Krebs-cycle pathways. SDHA levels were decreased in ALD patient-derived fibroblasts (Figure 2) as well as in AMPK $\alpha 1$-deleted Abcd1-KO mixed glial cells (Figure 3). Despite reduced SDHA expression (Figure 2), AMN patient-derived cells had mitochondrial OCR and ATP levels comparable to healthy controls [9]. This may be attributed to the fact that expression of complex $\mathrm{V}$ subunit (ATP-synthase subunit) was not significantly altered between AMN and healthy controls (Figure 2). Complex V subunit expression was decreased in ALD patient-derived cells (Figure 2) and in AMPK $\alpha 1$-deleted Abcd1-KO mice mixed glial cells (Figure 3). Deficiency of the complex V subunit ATP5A1 is associated with fatal neonatal encephalopathy [29]. 


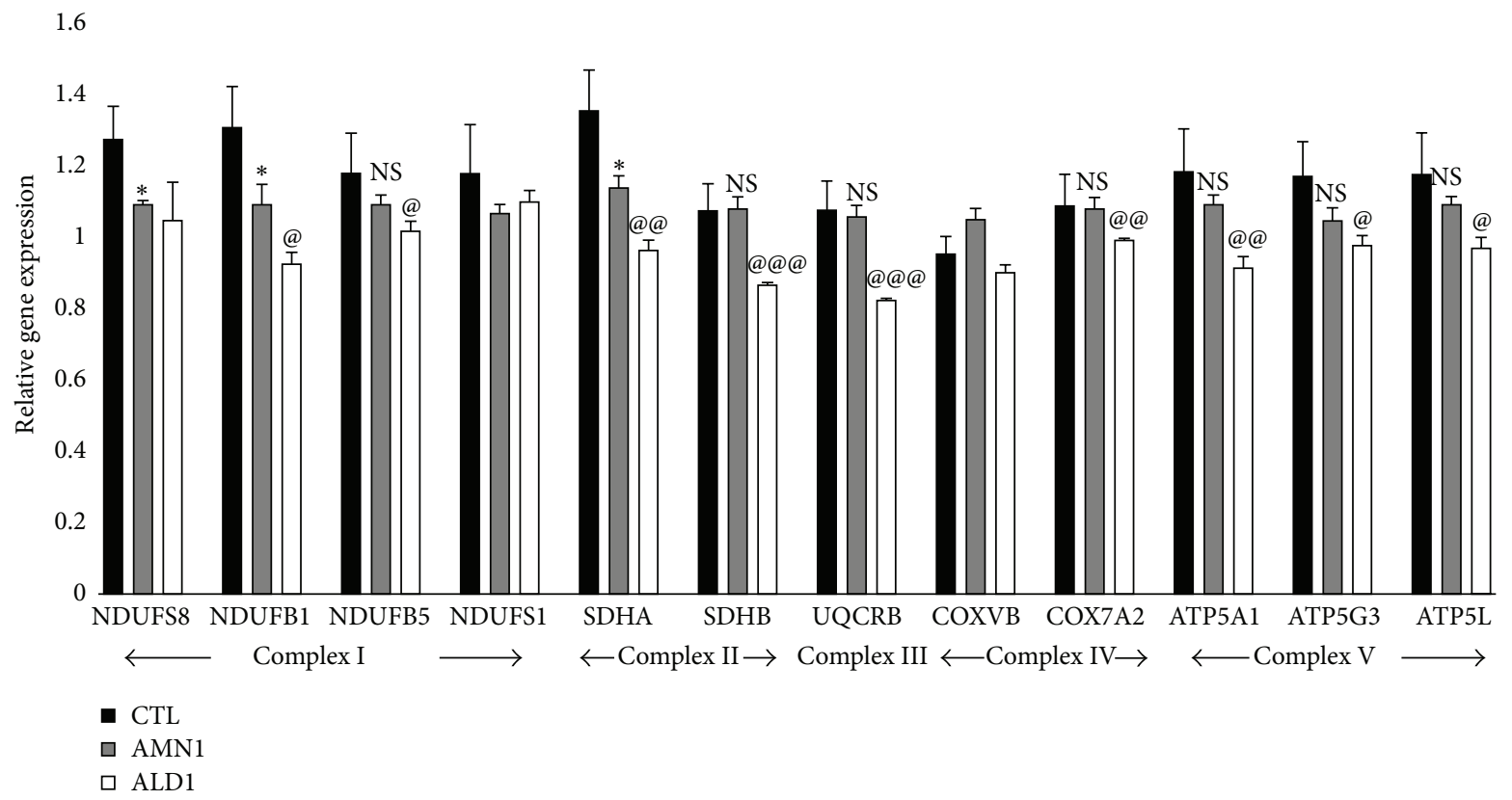

(a)
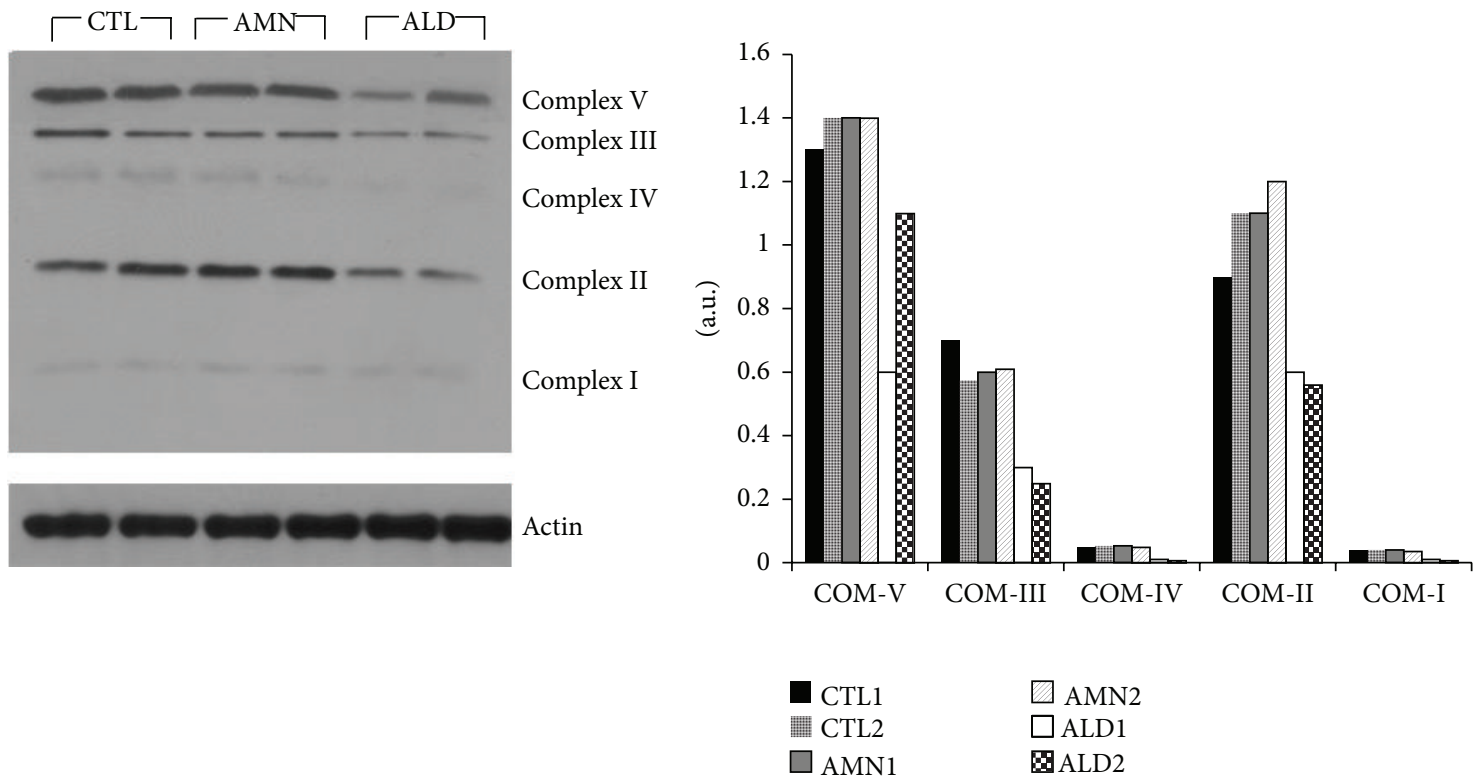

(b)

(c)

FIGURE 2: Mitochondrial complex subunit expression and levels in healthy control, adrenomyeloneuropathy (AMN), and adrenoleukodystrophy (ALD) patient-derived fibroblasts. Primary patient-derived skin fibroblasts from healthy control (CTL), AMN, and ALD were cultured as described in Section 2. mRNA (a) and protein (b) levels of mitochondrial complex subunits were significantly reduced in ALD patientderived fibroblasts. (c) Densitometric ratio of mitochondrial subunit levels versus actin in western blots. Results represent the mean \pm SD of triplicates from two different experiments. ${ }^{@} P<0.05$, ${ }^{@} P<0.01$, and ${ }^{@ @ @ ~} P<0.001$ ALD compared with AMN. ${ }^{*} P<0.05$ AMN compared with CTL. COM: complex, NS: nonsignificant.

AMPK affects mitochondrial biogenesis principally acting through peroxisome PGC- $1 \alpha[15,30,31]$. AMPK regulates both the PGC- $1 \alpha$ activation (phosphorylation) and its transcription [30, 31]. PGC- $1 \alpha$ in turn drives mitochondrial biogenesis through multiple mitochondrial transcription factors $[30,31]$. PGC- $1 \alpha$ levels were similar between WT and
Abcd1-KO mice mixed glial cells (Figure 4(a)). Knockdown of AMPK $\alpha 1$ significantly decreased $(P<0.001)$ PGC- $1 \alpha$ expression and levels in Abcd1-KO mice mixed glial cells (Figure 4(a)). Indeed, PGC- $1 \alpha$ levels were also decreased in vivo in AMPK-KO mice [15]. Whether the underexpression of mitochondrial complex subunits in ALD patient fibroblasts 


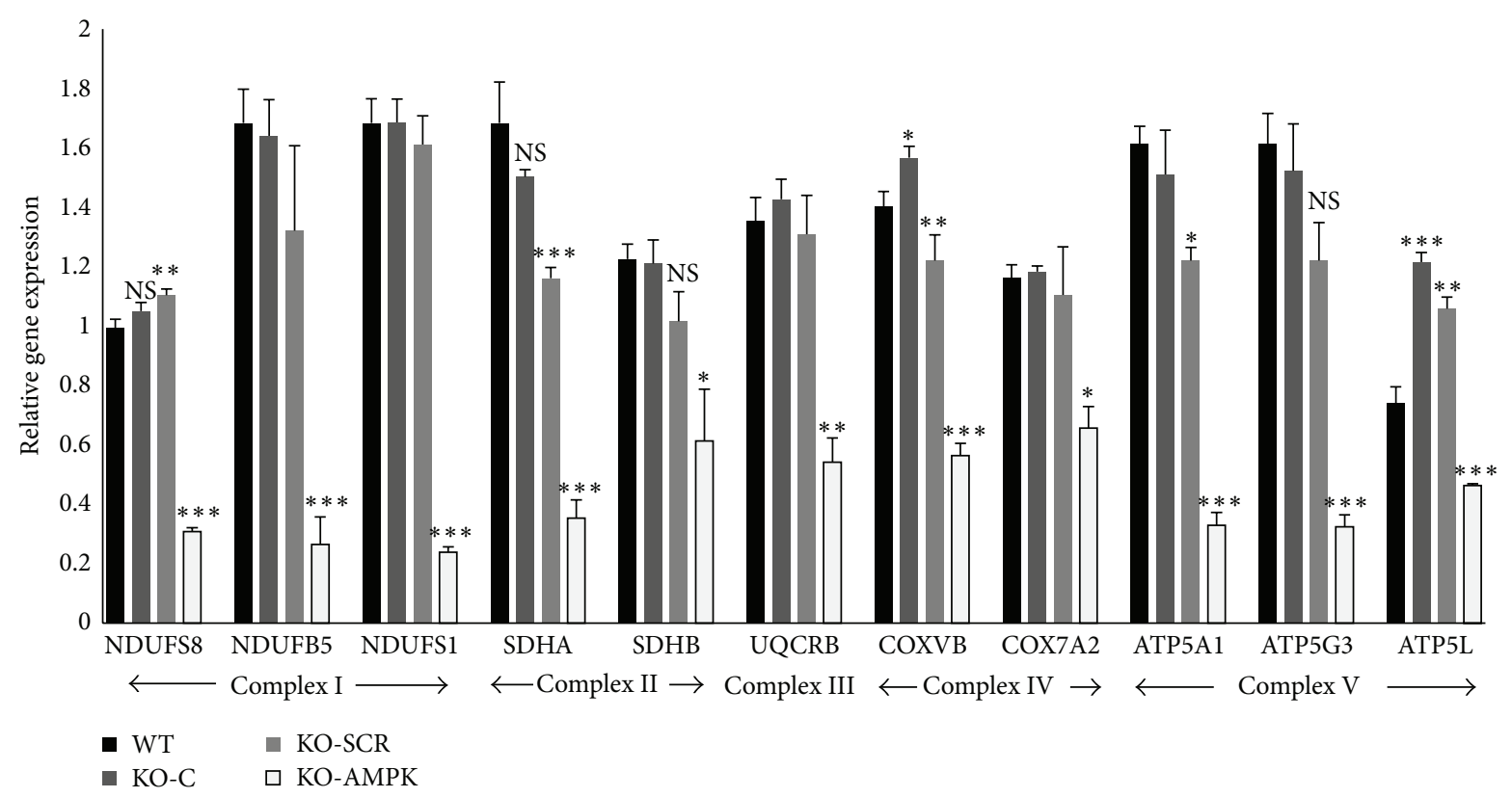

KO-C $\quad \square$ KO-AMPK

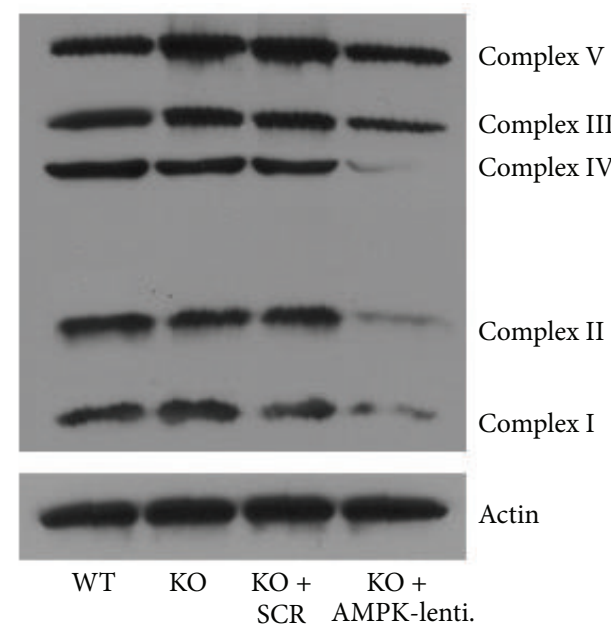

SCR AMPK-lenti.

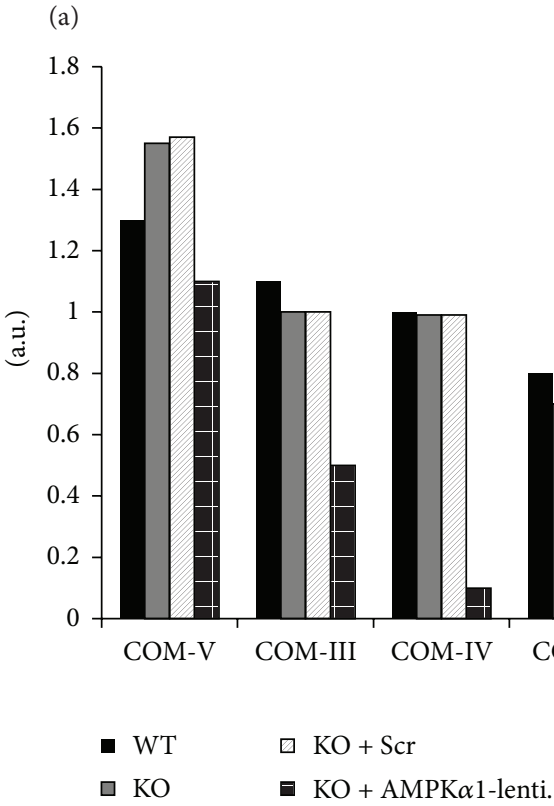

(c)

Figure 3: Mitochondrial complex subunit expression and levels in wild type, Abcdl-knockout (KO), and adenosine monophosphate activated protein kinase- (AMPK $\alpha 1-)$ deleted Abcd1-KO primary mixed glial cells. Wild type (WT) and Abcd1-KO primary mixed glial cells were cultured as described in Section 2. Abcd1-KO mixed glial cells were silenced for scrambled control (Scr) or AMPK $\alpha 1$ as described in Section 2. mRNA (a) and protein (b) levels of complex subunits were significantly reduced in Abcd1-KO mixed glial cells deleted for AMPK $\alpha 1$. (c) Densitometric ratio of mitochondrial subunit levels versus actin western blots. Results represent the mean \pm SD of triplicates from two different experiments. ${ }^{*} P<0.05 ;{ }^{* *} P<0.01 ;{ }^{* * *} P<0.001$. COM: complex, NS: nonsignificant.

is due to loss of AMPK, mutations in genes encoding the mitochondrial subunits, or a combination of both remains to be investigated.

3.3. Mitochondrial OCR Is Comparable between WT and Abcd1-KO Mixed Glial Cells and Is Significantly Reduced

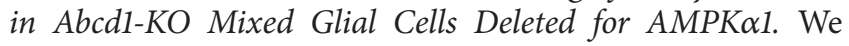

recently documented that mitochondrial OCR, a measure of mitochondrial OXPHOS, was significantly reduced in ALD (but not AMN) patient-derived cells [9]. These ALD patientderived cells also had a loss of AMPK and decreased levels of mitochondrial complex (I-V) subunit genes and proteins (Figures 2(a)-2(b)). Having demonstrated that AMPK $\alpha 1$ levels are similar between WT and Abcd1-KO mice mixed 


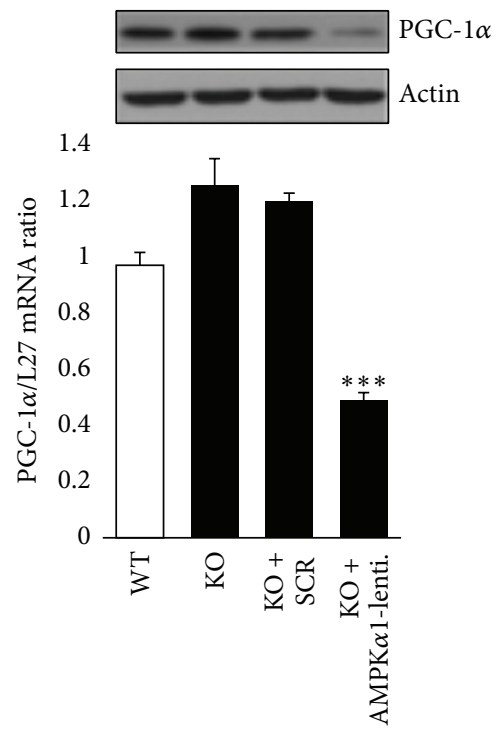

(a)

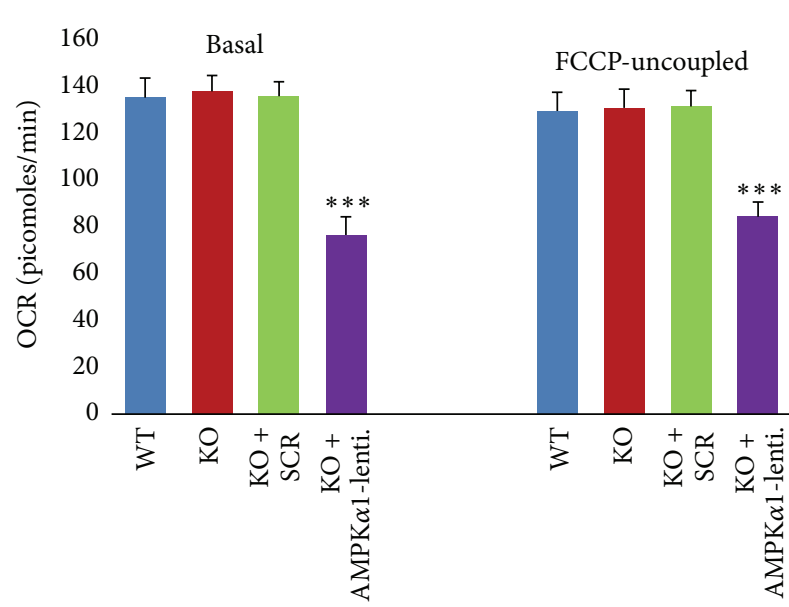

(c)

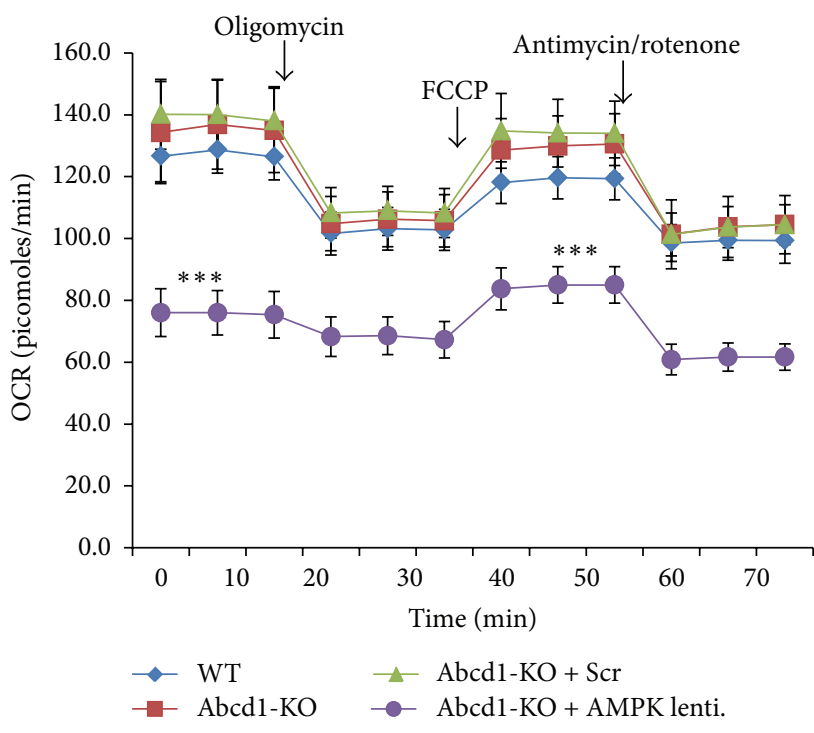

(b)

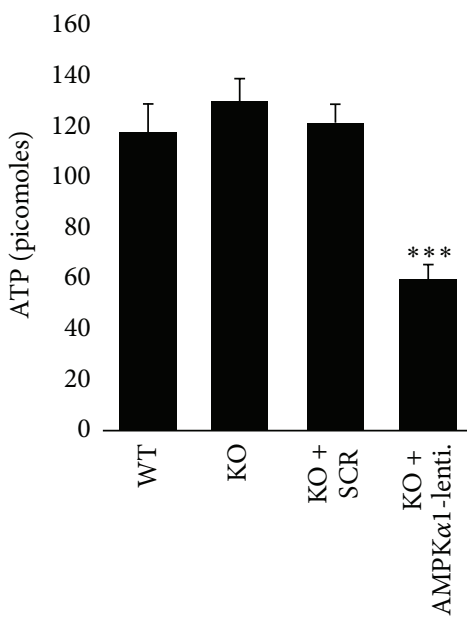

(d)

FIGURE 4: Mitochondrial bioenergetics in adenosine monophosphate activated protein kinase- (AMPK $\alpha 1$-) deleted Abcd1-knockout (KO) primary mixed glial cells. (a) Proliferator-activated receptor gamma coactivator 1-alpha (PGC-1 $\alpha$ ) protein and mRNA levels were significantly reduced in Abcd1-KO cells when deleted for AMPK $\alpha 1$. (b) Abcd1-KO mice mixed glial cells $\left(1.5 \times 10^{4}\right.$ cells/well) were plated in XF ${ }^{96} \mathrm{~V}^{3-P S}$ cell culture plates (Seahorse Bioscience) and deleted for AMPK $\alpha 1$ as described in Section 2. Mitochondrial oxygen consumption rate (OCR) was measured by sequential addition of oligomycin, FCCP, and antimycin/rotenone to measure basal and FCCP-uncoupled OCR. (c) Basal and FCCP-linked OCR was similar between wild type (WT) and Abcd1-KO mixed glial cells but decreased in Abcd1-KO mixed glial cells deleted for AMPK $\alpha$ 1. (d) A parallel 96-well plate was used for adenosine triphosphate (ATP) measurement. Results represent the mean \pm SD of triplicates from three different experiments. ${ }^{* * *} P<0.001$. NS: nonsignificant.

glial cells and that deletion of AMPK $\alpha 1$ significantly reduces mitochondrial complex (I-V) subunit expression and protein levels (Figure 3), we next characterized the bioenergetics of these cells using a Seahorse extracellular flux $\left(\mathrm{XF}^{\mathrm{e}}\right.$ 96, Seahorse Bioscience) analyzer. In this system mitochondrial respiration (OCR) is used to measure OXPHOS in intact cells [32]. WT and Abcd1-KO mice mixed glial cells $(1.5 \times$ $10^{4} /$ well) were plated in a $\mathrm{XF}^{\mathrm{e}} 96$-well microplate (Seahorse Bioscience). Abcd1-KO cells were deleted for AMPK $\alpha 1$ within the 96-well plate and OCR was measured 48 hours after AMPK $\alpha 1$ deletion (Figure 4(b)). Basal and FCCP-uncoupled maximal OCR (a measure of mitochondrial integrity [9]) were similar in WT and Abcd1-KO mice mixed glial cells (Figures 4(b) and 4(c)). Scramble (Scr) silencing did not impact the basal and FCCP-uncoupled OCR values between control and Scr silenced Abcd1-KO mixed glial cells (Figures $4(\mathrm{~b})$ and 4(c)). On the other hand, deletion of AMPK $\alpha 1$ significantly $(P<0.001)$ reduced both the basal and FCCPuncoupled OCR levels in Abcdl-KO mixed glial cells (Figures 4(b) and 4(c)). This provides evidence of a direct causal role for AMPK $\alpha 1$ in mitochondrial dysfunction in ALD, which was recently postulated by us in ALD patient-derived cells [9]. 


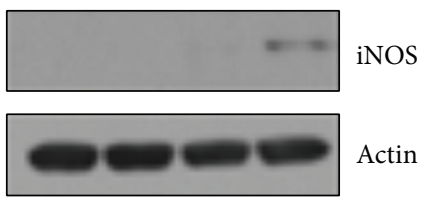

(a)

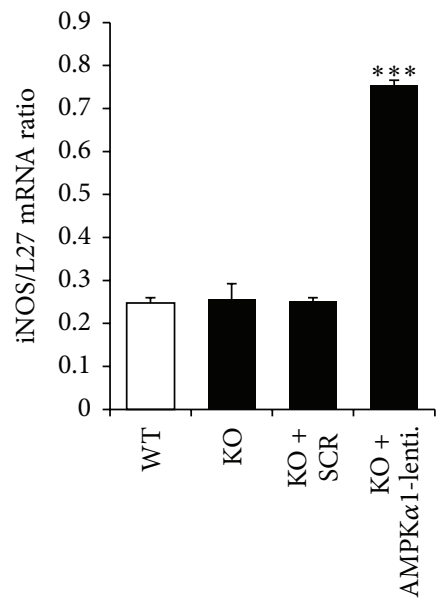

(b)

FIGURE 5: Adenosine monophosphate activated protein kinase- (AMPK $\alpha 1-$ ) deletion induces a spontaneous inflammatory response in Abcd1KO mixed glial cells. Abcd1-KO mice primary mixed glial cells were plated in 6-well plates and deleted for AMPK $\alpha 1$ using lentiviral vector. Inducible nitric oxide synthase (iNOS) protein (a) and mRNA levels (b) were induced in Abcd1-KO mixed glial cells when deleted for AMPK $\alpha 1$ using lentiviral particles. Results represent the mean $\pm \mathrm{SD}$ of triplicates from three different experiments. ${ }^{* * *} P<0.001$.

Mitochondrial OXPHOS generates the ATP energy for the cell [33]. Downregulation of complex V may affect the synthesis of ATP and ATP-dependent processes [33]. Therefore, the decreased expression of complex $\mathrm{V}$ subunits in ALD patient-derived cells observed in this study (Figure 2) supports our recent report of decreased ATP levels in the same patient-derived cells [9]. Abcd1-KO mice mimic human AMN phenotype, and primary mixed glial cells from AbcdlKO mice (compared with WT mixed glial cells) did not exhibit a decrease in ATP levels (Figure 4(d)) similar to our observation in human AMN patient-derived cells [9]. However, deletion of AMPK $\alpha 1$ led to a significant decrease $(P<0.001)$ in ATP levels in Abcdl-KO mice mixed glial cells (Figure 4(d)) in line with our observation of decreased complex V levels (Figure 3) and mitochondrial OCR (Figures 4(b) and 4(c)) in these cells. These findings, together with our recent report of differential loss of AMPK $\alpha 1$ in ALD [9], indicate a detrimental role for loss of AMPK $\alpha 1$ in inducing mitochondrial dysfunction in severe ALD pathology.

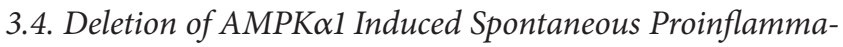
tory Response in Unstimulated Abcd1-KO Mice Mixed Glial Cells. The mechanism of the neuroinflammatory response in X-ALD remains unknown. In vitro loss of peroxisomal transporters $(\mathrm{Abcd} 2$ and $\mathrm{Abcd} 3)$ that have significant homology to the Abcdl gene has been shown to induce an inflammatory response in central nervous system cells [4]. However, levels of $A b c d 2$ and $A b c d 3$ are unaltered in the central nervous systems of X-ALD patients and, therefore, are likely not the disease modifying genes for initiation and progression of X-ALD [34]. Since AMPK $\alpha 1$ levels were reduced only in ALD patient-derived cells that presented with increased expression of proinflammatory genes [9], this provides a causal association between AMPK $\alpha 1$ loss and induction of the proinflammatory response in the ALD phenotype. This is expected since AMPK $\alpha 1$ is crucial for the anti-inflammatory skewing of cells $[10,19]$ and is involved in inhibiting lipid-induced inflammation [18]. Furthermore, AMPK-knockout animal models consistently demonstrate increased proinflammatory skewing [35]. To provide a direct evidence for this causal association between AMPK $\alpha 1$ loss and development of the proinflammatory response in X$A L D$, we investigated the expression and levels of inducible nitric oxide synthase (iNOS) in Abcd1-KO mixed glial cells deleted for AMPK $\alpha 1$ (Figure 5). In vivo [4] and in vitro [16] evidence implicates involvement of iNOS in X-ALD neuropathology. Basal iNOS levels were undetectable in WT and Abcdl-KO mixed glial cells (Figure 5). Silencing with control (Scr) lentiviral particles did not induce any iNOS level (Figure 5). However, AMPK $\alpha 1$ deletion significantly induced $(P<0.001)$ the iNOS level (Figure 5(a)) and gene expression (Figure 5(b)) in unstimulated Abcd1-KO mixed glial cells.

\section{Conclusions}

In conclusion, these findings represent the first direct evidence of a link between loss of AMPK $\alpha 1$ and initiation/augmentation of mitochondrial dysfunction and the neuroinflammatory response in X-ALD, especially in mixed glial cells. The central nervous system (brain and spinal cord) is the target organ for development of X-ALD therapies. AMPK $\alpha 1$, therefore, provides a novel target for development of therapeutic strategies aimed at ameliorating the initiation and/or progression of the neuroinflammatory response in $\mathrm{X}$ ALD.

\section{Conflict of Interests}

The authors declare that there is no conflict of interests regarding the publication of this paper. 


\section{Acknowledgments}

The authors thank Stephanie Stebens for editing the paper. This study was supported by funds from Henry Ford Health System Proposal Development Grant (A30916) and Mentored Scientist Grant (A10263) to Jaspreet Singh.

\section{References}

[1] S. Kemp, J. Berger, and P. Aubourg, "X-linked adrenoleukodystrophy: clinical, metabolic, genetic and pathophysiological aspects," Biochimica et Biophysica Acta, vol. 1822, no. 9, pp. 14651474, 2012.

[2] M. Contreras, J. Mosser, J. L. Mandel, P. Aubourg, and I. Singh, "The protein coded by the X-adenoleukodystrophy gene is a peroxisomal integral membrane protein," FEBS Letters, vol. 344, no. 2-3, pp. 211-215, 1994.

[3] M. Engelen, S. Kemp, M. de Visser et al., "X-linked adrenoleukodystrophy (X-ALD): clinical presentation and guidelines for diagnosis, follow-up and management," Orphanet Journal of Rare Diseases, vol. 7, no. 1, article 51, 2012.

[4] I. Singh and A. Pujol, "Pathomechanisms underlying Xadrenoleukodystrophy: a three-hit hypothesis," Brain Pathology, vol. 20, no. 4, pp. 838-844, 2010.

[5] J.-F. Lu, A. M. Lawler, P. A. Watkins et al., "A mouse model for X-linked adrenoleukodystrophy," Proceedings of the National Academy of Sciences of the United States of America, vol. 94, no. 17, pp. 9366-9371, 1997.

[6] T. Kobayashi, N. Shinnoh, A. Kondo, and T. Yamada, "Adrenoleukodystrophy protein-deficient mice represent abnormality of very long chain fatty acid metabolism," Biochemical and Biophysical Research Communications, vol. 232, no. 3, pp. 631636, 1997.

[7] S. Forss-Petter, H. Werner, J. Berger et al., "Targeted inactivation of the X-linked adrenoleukodystrophy gene in mice," Journal of Neuroscience Research, vol. 50, no. 5, pp. 829-843, 1997.

[8] A. Pujol, C. Hindelang, N. Callizot, U. Bartsch, M. Schachner, and J. L. Mandel, "Late onset neurological phenotype of the $\mathrm{X}$-ALD gene inactivation in mice: a mouse model for adrenomyeloneuropathy," Human Molecular Genetics, vol. 11, no. 5, pp. 499-505, 2002.

[9] J. Singh and S. Giri, "Loss of AMP-activated protein kinase in $\mathrm{X}$-linked adrenoleukodystrophy patient-derived fibroblasts and lymphocytes," Biochemical and Biophysical Research Communications, vol. 445, no. 1, pp. 126-131, 2014.

[10] K. C. Carroll, B. Viollet, and J. Suttles, "AMPKalphal deficiency amplifies proinflammatory myeloid APC activity and CD40 signaling," Journal of Leukocyte Biology, vol. 94, no. 6, pp. 11131121, 2013.

[11] N. Nath, M. Khan, R. Rattan et al., "Loss of AMPK exacerbates experimental autoimmune encephalomyelitis disease severity," Biochemical and Biophysical Research Communications, vol. 386, no. 1, pp. 16-20, 2009.

[12] L. A. J. O'Neill and D. Grahame Hardie, "Metabolism of inflammation limited by AMPK and pseudo-starvation," Nature, vol. 493, no. 7432, pp. 346-355, 2013.

[13] N. Nath, M. Khan, M. K. Paintlia, I. Singh, M. N. Hoda, and S. Giri, "Metformin attenuated the autoimmune disease of the central nervous system in animal models of multiple sclerosis," The Journal of Immunology, vol. 182, pp. 8005-8014, 2009.
[14] N. Nath, S. Giri, R. Prasad, M. L. Salem, A. K. Singh, and I. Singh, "5-Aminoimidazole-4-carboxamide ribonucleoside: a novel immunomodulator with therapeutic efficacy in experimental autoimmune encephalomyelitis," The Journal of Immunology, vol. 175, no. 1, pp. 566-574, 2005.

[15] H. M. O’Neill, S. J. Maarbjerg, J. D. Crane et al., "AMP-activated protein kinase (AMPK) betalbeta2 muscle null mice reveal an essential role for AMPK in maintaining mitochondrial content and glucose uptake during exercise," Proceedings of the National Academy of Sciences of the United States of America, vol. 108, no. 38, pp. 16092-16097, 2011.

[16] J. Singh, M. Khan, and I. Singh, "Silencing of Abcd1 and Abcd2 genes sensitizes astrocytes for inflammation: implication for Xadrenoleukodystrophy," Journal of Lipid Research, vol. 50, no. 1, pp. 135-147, 2009.

[17] N. Nath, M. Khan, R. Rattan et al., "Loss of AMPK exacerbates experimental autoimmune encephalomyelitis disease severity," Biochemical and Biophysical Research Communications, vol. 386, no. 1, pp. 16-20, 2009.

[18] S. Galic, M. D. Fullerton, J. D. Schertzer et al., "Hematopoietic AMPK $\beta 1$ reduces mouse adipose tissue macrophage inflammation and insulin resistance in obesity," Journal of Clinical Investigation, vol. 121, no. 12, pp. 4903-4915, 2011.

[19] R. Mounier, M. Théret, L. Arnold et al., "AMPK $\alpha 1$ regulates macrophage skewing at the time of resolution of inflammation during skeletal muscle regeneration," Cell Metabolism, vol. 18, no. 2, pp. 251-264, 2013.

[20] J. Singh, M. Khan, A. Pujol, M. Baarine, and I. Singh, "Histone deacetylase inhibitor upregulates peroxisomal fatty acid oxidation and inhibits apoptotic cell death in abcdl-deficient glial cells," PLoS ONE, vol. 8, no. 7, Article ID e70712, 2013.

[21] S. Fourcade, J. López-Erauskin, M. Ruiz, I. Ferrer, and A. Pujol, "Mitochondrial dysfunction and oxidative damage cooperatively fuel axonal degeneration in X-linked adrenoleukodystrophy," Biochimie, vol. 98, no. 1, pp. 143-149, 2014.

[22] J. Berger, S. Forss-Petter, and F. S. Eichler, "Pathophysiology of X-linked adrenoleukodystrophy," Biochimie, vol. 98, no. 1, pp. 135-142, 2014.

[23] L. De Meirleir, S. Seneca, W. Lissens et al., "Respiratory chain complex V deficiency due to a mutation in the assembly gene ATP12," Journal of Medical Genetics, vol. 41, no. 2, pp. 120-124, 2004.

[24] X. Zhu, X. Peng, M. X. Guan, and Q. Yan, "Pathogenic mutations of nuclear genes associated with mitochondrial disorders," Acta Biochimica et Biophysica Sinica, vol. 41, no. 3, pp. 179-187, 2009.

[25] R. H. Triepels, L. P. van den Heuvel, J. L. C. M. Loeffen et al., "Leigh syndrome associated with a mutation in the NDUFS7 (PSST) nuclear encoded subunit of complex I," Annals of Neurology, vol. 45, no. 6, pp. 787-790, 1999.

[26] J. Smeitink and L. van den Heuvel, "Human mitochondrial complex I in health and disease," The American Journal of Human Genetics, vol. 64, no. 6, pp. 1505-1510, 1999.

[27] P. Bénit, D. Chretien, N. Kadhom et al., "Large-scale deletion and point mutations of the nuclear NDUFV1 and NDUFS1 genes in mitochondrial complex I deficiency," American Journal of Human Genetics, vol. 68, no. 6, pp. 1344-1352, 2001.

[28] T. Bourgeron, P. Rustin, D. Chretien et al., "Mutation of a nuclear succinate dehydrogenase gene results in mitochondrial respiratory chain deficiency," Nature Genetics, vol. 11, no. 2, pp. 144-149, 1995. 
[29] A. I. Jonckheere, G. Herma Renkema, M. Bras et al., "A complex v ATP5A1 defect causes fatal neonatal mitochondrial encephalopathy," Brain, vol. 136, no. 5, pp. 1544-1554, 2013.

[30] P. J. Fernandez-Marcos and J. Auwerx, "Regulation of PGClalpha, a nodal regulator of mitochondrial biogenesis," The American Journal of Clinical Nutrition, vol. 93, no. 4, pp. 884S890S, 2011.

[31] R. C. Scarpulla, "Metabolic control of mitochondrial biogenesis through the PGC-1 family regulatory network," Biochimica et Biophysica Acta, vol. 1813, no. 7, pp. 1269-1278, 2011.

[32] M. B. de Moura and B. van Houten, "Bioenergetic analysis of intact mammalian cells using the seahorse XF24 extracellular flux analyzer and a luciferase ATP assay," in Molecular Toxicology Protocols, vol. 1105 of Methods in Molecular Biology, pp. 589602, Humana Press, 2014.

[33] F. Reinecke, J. A. M. Smeitink, and F. H. van der Westhuizen, "OXPHOS gene expression and control in mitochondrial disorders," Biochimica et Biophysica Acta, vol. 1792, no. 12, pp. 11131121, 2009.

[34] T. Matsukawa, M. Asheuer, Y. Takahashi et al., "Identification of novel SNPs of ABCD1, ABCD2, ABCD3, and ABCD4 genes in patients with X-linked adrenoleukodystrophy (ALD) based on comprehensive resequencing and association studies with ALD phenotypes," Neurogenetics, vol. 12, no. 1, pp. 41-50, 2011.

[35] B. Viollet, Y. Athea, R. Mounier et al., "AMPK: lessons from transgenic and knockout animals," Frontiers in Bioscience (Landmark Ed), vol. 14, no. 1, pp. 19-44, 2009. 


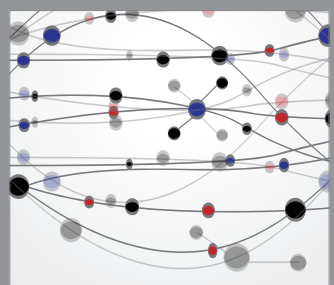

The Scientific World Journal
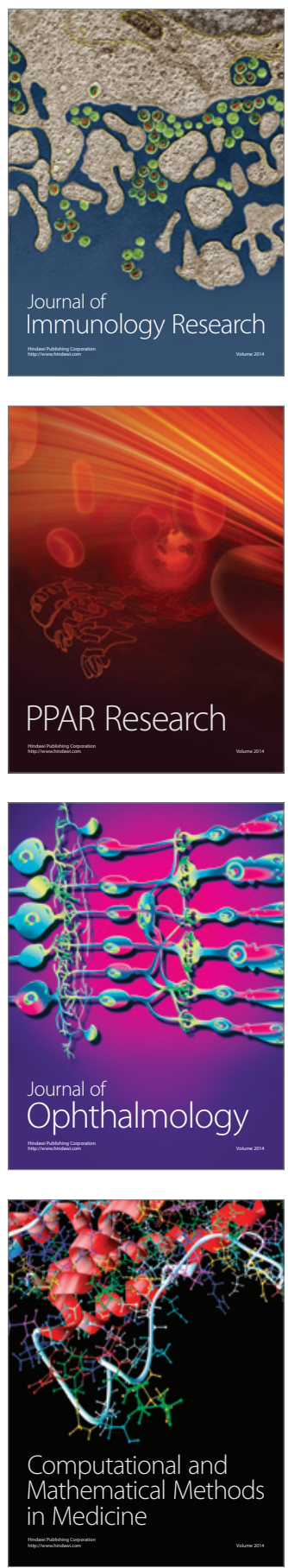

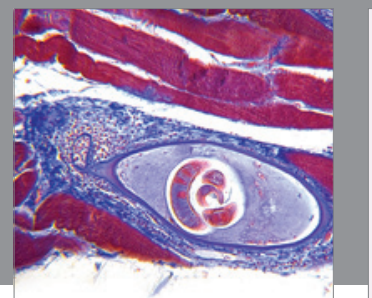

Gastroenterology

Research and Practice
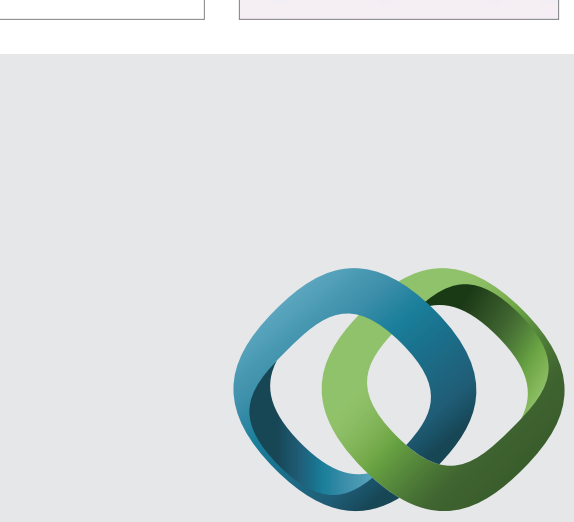

\section{Hindawi}

Submit your manuscripts at

http://www.hindawi.com
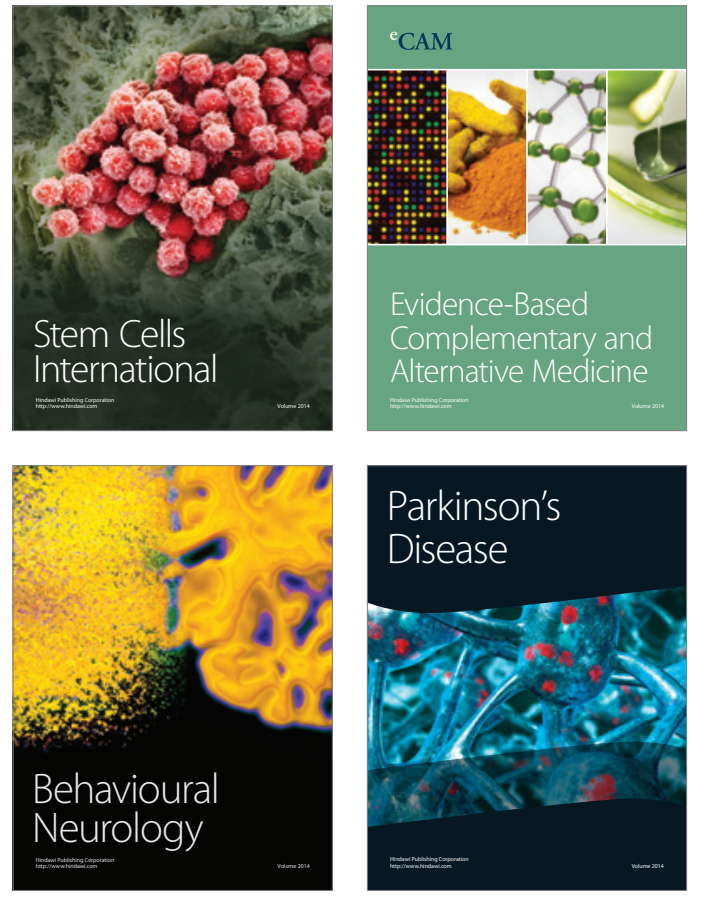
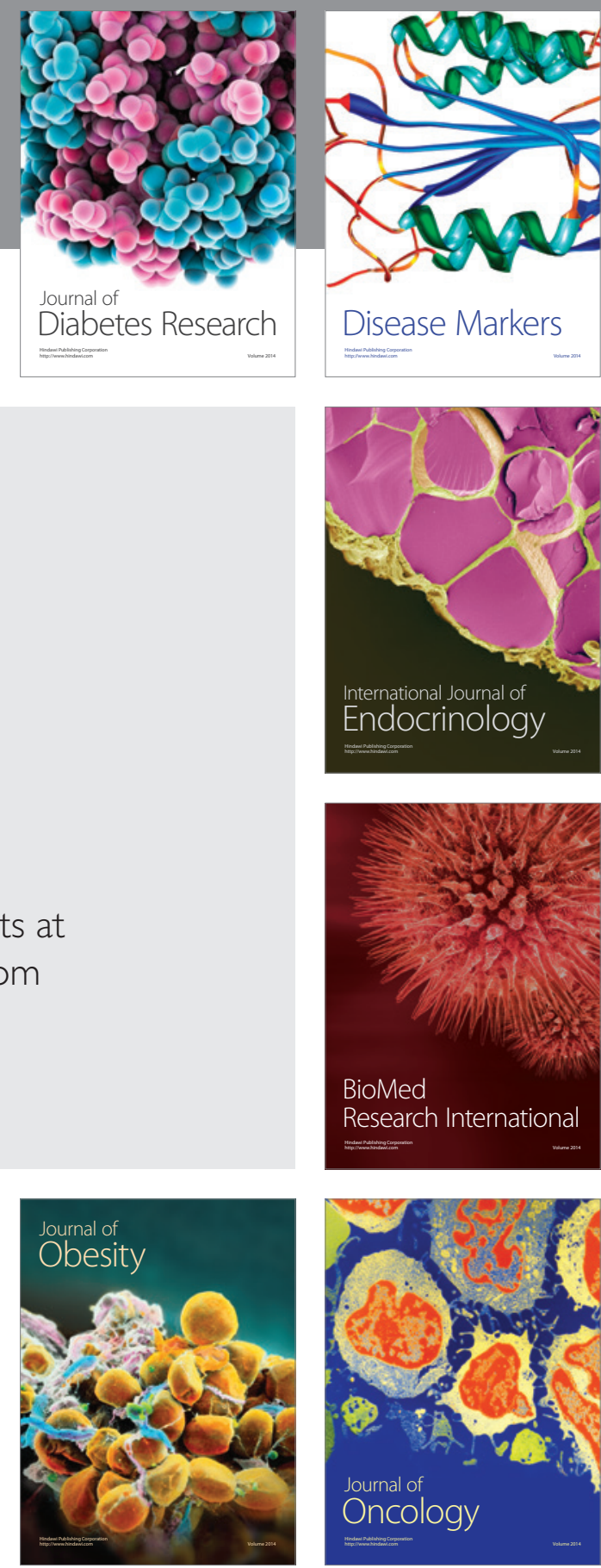

Disease Markers
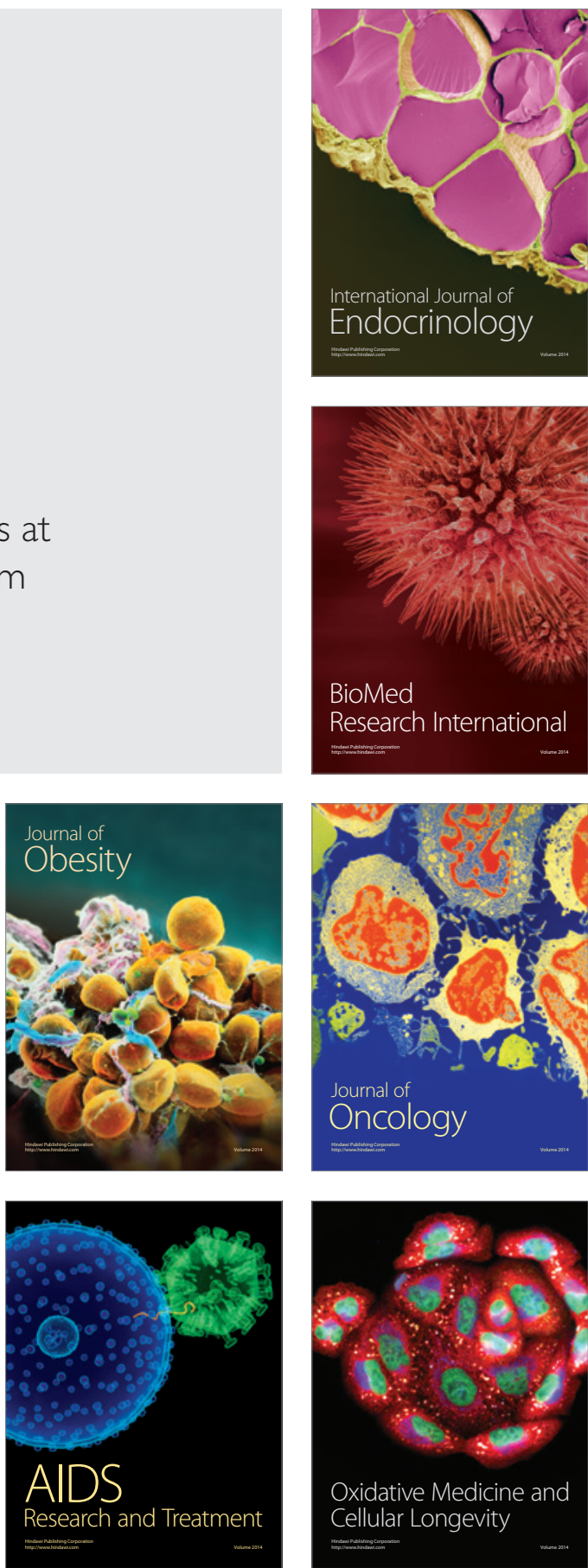